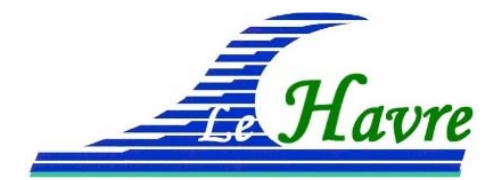

XVI'̀mes Journées Nationales Génie Côtier - Génie Civil

Le Havre, 2020

DOI:10.5150/jngcgc.2020.084 C Editions Paralia CFL

disponible en ligne - http://www.paralia.fr - available online

\title{
Évaluation du nombre et de la valeur des logements menacés par le recul du trait de côte au cours du $21^{\text {ème }}$ siècle
}

\author{
François HEDOU ${ }^{1}$, Boris LECLERC ${ }^{1}$
}

\author{
1. Cerema, direction Eau, mer et fleuves, 155, rue Pierre Bouguer - BP5, \\ 29280 Plouzané, France. \\ francois.hedou@cerema.fr ; boris.leclerc@cerema.fr
}

\section{Résumé :}

À la demande du ministère de la Transition écologique et solidaire, le Cerema a étudié à l'échelle nationale (métropole et DROM) le nombre et la valeur des logements potentiellement atteints par le recul du trait de côte au cours de ce siècle. Un trait de côte composite a été créé en assemblant principalement les données issues de "l'indicateur national de l'érosion côtière" et de "la cartographie nationale des ouvrages et aménagements littoraux" (position et typologie des ouvrages), constituées par le Cerema dans le cadre de la Stratégie nationale de gestion intégrée du trait de côte. Afin de combler l'absence ponctuelle de connaissance de recul historique, notamment au droit des ouvrages, des taux d'évolution dits "régionaux" ont été calculés par secteur homogène en combinant plusieurs paramètres : géomorphologie, géologie et découpage du littoral en unités sédimentaires. Ce document expose plus en détail cette étape essentielle à ces travaux. Des hypothèses simplificatrices sur l'évolution du trait de côte et sur le maintien ou non des ouvrages de protection ont été formulées afin de projeter annuellement la position du trait de côte jusqu'en 2100 selon 6 scénarios. L'exploitation à l'échelle nationale des bases de données des "Fichiers fonciers" et de "DV3F", constituées par le Cerema à partir d'informations fiscales, a permis d'estimer le nombre et la valeur des logements proches du littoral. Le croisement entre les projections du trait de côte et les logements montre qu'il existe globalement un facteur 10 entre le scénario le plus favorable et le plus défavorable avec des pertes cumulées de logements en France estimés entre 0,8 et 8 milliards d'Euros à l'échéance 2100. Ces estimations à des échéances lointaines comprennent certes de nombreuses limites méthodologiques et incertitudes, mais elles apportent un premier éclairage national aux réflexions en cours sur l'indispensable adaptation des territoires littoraux aux conséquences du dérèglement climatique.

\section{Mots-clés :}

Recul du trait de côte, Littoral, Enjeux, Logement, Etude prospective, Etude nationale 


\section{Thème 7 - Risques côtiers}

\section{Introduction}

Toutes les régions françaises sont concernées, à des degrés divers, par le recul du trait de côte qui affecte $20 \%$ du linéaire côtier (HÉDOU et al., 2018). Des bâtiments sont régulièrement atteints par la mer et de nombreux autres sont potentiellement menacés, d'autant plus que la hausse du niveau marin induite par le dérèglement climatique pourrait aggraver les risques littoraux. Dans le cadre des réflexions nationales sur les mesures d'adaptation à prendre sur le littoral et plus particulièrement de leur dimensionnement financier, le ministère de la Transition écologique et solidaire a confié au Cerema l'étude du nombre et de la valeur des logements potentiellement atteints par le recul du trait de côte au cours de ce siècle à l'échelle nationale (métropole et DROM).

\section{Données principales pour le tracé et les caractéristiques du trait de côte}

Une position précise et datée d'un trait de côte couvrant l'ensemble du littoral doit être définie dès le début de l'étude. Celui-ci est composé de parties naturelles et de secteurs artificialisés. C'est à partir de cette ligne composite que seront projetées les positions théoriques et futures du trait de côte, elle constitue ainsi la situation de référence. D'autres informations sont ensuite associées à cette donnée.

\subsection{Trait de côte "naturel"}

La position du trait de côte "naturel" a été relevée à partir de la photo-interprétation de supports orthophotographiques dans le cadre l'indicateur national de l'érosion côtière (CEREMA \& MTES, 2018). Son linéaire cumulé est de $6132 \mathrm{~km}$ (4 $572 \mathrm{~km}$ en métropole et $1560 \mathrm{~km}$ dans les DROM). Cette position est datée selon les supports exploités.

\subsection{Trait de côte avec ouvrages}

Les éléments ayant servi à constituer la cartographie nationale des ouvrages et aménagements littoraux (CEREMA \& MTES, 2017) sont exploités. Seuls les ouvrages encore visibles sur les dernières photographies aériennes et qui présentent une orientation longitudinale (perrés, digues, murs, etc.) sont pris en compte pour cette étude. Ils représentent un linéaire cumulé de 1421 km (1 269 km en métropole et 152 km en outremer).

\subsection{Nature du trait de côte}

Les informations sur la géologie et la géomorphologie du trait de côte issue du projet européen Eurosion (CE, 2004), sont complétées par le Cerema pour la Martinique, Mayotte et la Réunion. Produites sur un tracé différent, ces données sont affectées (au plus proche voisin) aux parties du trait de côte "naturel" et à ouvrages préalablement découpées en section respectivement longues de 50 et de 100 mètres de long (maximum). 


\section{XVI èmes Journées Nationales Génie Côtier - Génie Civil \\ Le Havre 2020}

\subsection{Unités morpho-sédimentaires}

Comme pour la nature du trait de côte, le découpage du littoral en une centaine d'unités morpho-sédimentaires (MEEM, 2016) est intégré au projet avec une affectation des informations au plus proche.

\section{Complétion des données pour l'évolution du trait de côte}

Les taux d'évolution du trait de côte ont été produits selon des profils disposés tous les 200 mètres le long du littoral dans le cadre de l'indicateur national de l'érosion côtière (CEREMA \& MTES, 2018). Ces données sont affectées (au plus proche voisin) aux parties du trait de côte "naturel". Certains secteurs du trait de côte composite ne disposent cependant d'aucune information quant à l'évolution du trait de côte du fait d'une impossibilité à calculer un taux d'évolution. Cette impossibilité résulte du caractère non exploitable de certaines orthophotographies (présence de nuage) ou de la présence d'ouvrages se substituant au trait de côte. Il est ainsi nécessaire d'interpoler les valeurs locales d'évolution du trait de côte pour les affecter aux secteurs manquants. Différentes stratégies décrites après ont été mises en œuvre.

\subsection{Ajout ponctuel d'informations par interpolation}

Afin de combler les manques ponctuels de taux d'évolution, aussi bien sur ses portions naturelles que sur celles artificielles (présence d'ouvrages longitudinaux), il est procédé à une interpolation des taux sur une faible distance. Celle-ci est réalisée dans un rayon de 1000 mètres autour du dernier segment du trait de côte "naturel" disposant d'une valeur d'évolution selon un maillage de $100 \times 100$ mètres.

\subsection{Calcul de taux "régionaux"}

L'interpolation n'étant pas suffisante pour fournir des données sur les taux d'évolution sur de larges secteurs possédant un trait de côte très artificialisés, des taux médians d'évolution dits "régionaux" sont alors calculés en combinant plusieurs paramètres : géomorphologie, géologie et découpage du littoral en unités morpho-sédimentaires (tableaux 1 et 2). Seules les valeurs en recul de l'indicateur national sont prises en compte pour ce calcul. L'évolution du littoral pouvant être très locale, il a été recherché un compromis entre, un découpage spatial fin permettant une régionalisation des taux d'évolution au plus près des spécificités locales, et un nombre suffisant de valeur en recul au sein de ces zones pour garantir un sens statistique aux valeurs retenues. Des regroupements d'unités morpho-sédimentaires sont ainsi réalisés pour les côtes rocheuses de Bretagne, de la région PACA et de Corse possédant peu de valeur en recul et localement en outre-mer. Les côtes à falaises composées de roches sédimentaire et plus sujettes au recul ont été distinguées. Les taux médians régionaux ainsi calculés sont enfin affectés aux segments du trait de côte "naturel" et à ouvrages selon leur géomorphologie et le cas échéant leur géologie pour les côtes à falaises. 


\section{Thème 7 - Risques côtiers}

Tableau 1. Taux médians régionaux calculés en métropole.

\begin{tabular}{|c|c|c|c|c|}
\hline \multirow[b]{2}{*}{ Nom de l'unité } & \multicolumn{4}{|c|}{ Taux médians régionaux (m/an) } \\
\hline & Unité & $\begin{array}{l}\text { Côtes basses } \\
\text { sableuses }\end{array}$ & \begin{tabular}{|l} 
Falaises de \\
roches \\
sédimentaires
\end{tabular} & \begin{tabular}{|l} 
Falaises (hors \\
roches \\
sédimentaires)
\end{tabular} \\
\hline Frontière belge à Sangatte & $-0,2$ & $-0,2$ & 0 & $-0,3$ \\
\hline Sangatte à Hardelot-Plage & $-0,2$ & $-0,7$ & $-0,1$ & $-0,2$ \\
\hline Hardelot-Plage à la pointe du Hourdel & $-0,3$ & $-0,4$ & 0 & 0 \\
\hline Pointe du Hourdel à Ault & $-0,6$ & $-0,6$ & 0 & 0 \\
\hline Ault à la pointe d'Antifer & $-0,2$ & $-0,1$ & $-0,2$ & $-0,1$ \\
\hline Pointe d'Antifer à Sainte-Adresse & $-0,1$ & 0 & $-0,1$ & 0 \\
\hline Sainte-Adresse à ST-Vaast la Hougue & $-0,2$ & $-0,2$ & $-0,2$ & 0 \\
\hline St-Vaast la Hougue à Barfleur & $-0,1$ & $-0,1$ & 0 & $-0,2$ \\
\hline Barfleur à la Hague & $-0,2$ & $-0,2$ & 0 & $-0,1$ \\
\hline Cap de la Hague à la pointe du Roc & $-0,4$ & $-0,4$ & $-0,2$ & $-0,1$ \\
\hline Estuaire externe de la Loire & $-0,1$ & $-0,1$ & 0 & 0 \\
\hline Baie de Bourgneuf & $-0,2$ & $-0,2$ & 0 & $-0,2$ \\
\hline Pointe de l'Herbaudière - Pointe de la Fosse & $-0,2$ & $-0,2$ & 0 & 0 \\
\hline Île d'Yeu & $-0,2$ & $-0,2$ & 0 & $-0,1$ \\
\hline Domaine Vendéen & $-0,2$ & $-0,2$ & $-0,1$ & $-0,1$ \\
\hline Pt du Grouin Les Pertuis dont Aix, Oléron et Ré & $-0,3$ & $-0,3$ & $-0,2$ & $-0,1$ \\
\hline Pertuis de Maumusson - Embouchure Gironde & $-4,9$ & $-5,3$ & $-0,5$ & 0 \\
\hline Estuaire de la Gironde & -3 & -3 & 0 & 0 \\
\hline Pte de la Négade - Pte du Cap Ferret & $-0,9$ & $-0,9$ & 0 & 0 \\
\hline Bassin d'Arcachon & $-0,1$ & $-2,4$ & 0 & 0 \\
\hline Arcachon - Pte Saint-Martin & $-0,3$ & $-0,3$ & $-0,3$ & 0 \\
\hline Côte rocheuse basque & $-0,2$ & $-0,1$ & $-0,2$ & $-0,4$ \\
\hline La côte Vermeille & $-0,2$ & $-0,2$ & 0 & $-0,2$ \\
\hline Le Roussillon & $-0,4$ & $-0,4$ & 0 & 0 \\
\hline Le Narbonnais & $-0,4$ & $-0,4$ & 0 & 0 \\
\hline Le lido de Sète & $-0,3$ & $-0,3$ & 0 & $-0,1$ \\
\hline Le golfe d'Aigues-Mortes & $-0,3$ & $-0,3$ & 0 & 0 \\
\hline La Camargue & $-1,9$ & $-1,9$ & $-0,1$ & 0 \\
\hline Miomo - Moriani & $-0,3$ & $-0,3$ & 0 & 0 \\
\hline Moriani - Solenzara & $-0,3$ & $-0,3$ & 0 & $-0,1$ \\
\hline Bretagne Nord & $-0,1$ & $-0,2$ & $-0,1$ & $-0,1$ \\
\hline Bretagne Sud & $-0,2$ & $-0,2$ & $-0,2$ & $-0,1$ \\
\hline Embouchure Nord de la Gironde & $-0,1$ & $-0,9$ & $-0,1$ & 0 \\
\hline PACA - rocheux & $-0,2$ & $-0,2$ & $-0,1$ & $-0,1$ \\
\hline Corse - rocheux & $-0,2$ & $-0,2$ & $-0,1$ & $-0,1$ \\
\hline
\end{tabular}




\section{XVIèmes Journées Nationales Génie Côtier - Génie Civil \\ Le Havre 2020}

Tableau 2. Taux médians régionaux calculés en outre-mer.

\begin{tabular}{|c|c|c|c|c|c|c|}
\hline \multirow[b]{2}{*}{ Territoire } & \multirow[b]{2}{*}{ Nom de l'unité } & \multicolumn{5}{|c|}{ Taux médians régionaux (m/an) } \\
\hline & & Unité & \begin{tabular}{|l} 
Côtes \\
basses \\
sableuses \\
\end{tabular} & \begin{tabular}{|l} 
Falaises de \\
roches \\
sédimentaires
\end{tabular} & $\begin{array}{l}\text { Falaises (hors } \\
\text { roches } \\
\text { sédimentaires) }\end{array}$ & Mangroves \\
\hline \multirow{9}{*}{ Guadeloupe } & Pointe Allegre - Pointe d'Antigues & $-0,3$ & $-0,3$ & 0 & $-0,4$ & $-0,2$ \\
\hline & Pointe d'Antigues - Pointe des Colibris & $-0,4$ & $-0,4$ & $-0,5$ & $-0,4$ & 0 \\
\hline & \begin{tabular}{|l}
$\begin{array}{l}\text { Pointe des Colibris - Pointe de la } \\
\text { Verdure }\end{array}$ \\
\end{tabular} & $-0,3$ & $-0,4$ & $-0,1$ & 0 & 0 \\
\hline & Pointe de la Verdure - Riviere a Goyave & $-0,2$ & $-0,2$ & $-0,2$ & 0 & $-0,2$ \\
\hline & Riviere a Goyave - Pointe du Vieux Fort & $-0,2$ & $-0,2$ & 0 & $-0,2$ & $-0,3$ \\
\hline & Pointe du Vieux Fort - Pointe Allegre & $-0,2$ & $-0,2$ & 0 & $-0,2$ & 0 \\
\hline & Les Saintes & $-0,2$ & $-0,3$ & 0 & $-0,1$ & 0 \\
\hline & Marie-Galante & $-0,2$ & $-0,2$ & $-0,1$ & $-0,1$ & 0 \\
\hline & La Désirade & $-0,3$ & $-0,7$ & $-0,3$ & 0 & 0 \\
\hline \multirow{3}{*}{ Martinique } & Embouchure Precheur - Ilet Lapin & $-0,2$ & $-0,3$ & $-0,1$ & $-0,4$ & $-0,1$ \\
\hline & Ilet Lapin - Pointe Borgnesse & $-0,2$ & $-0,2$ & $-0,2$ & 0 & $-0,3$ \\
\hline & $\begin{array}{l}\text { Pointe Borgnesse - Embouchure } \\
\text { Precheur }\end{array}$ & $-0,2$ & $-0,2$ & $-0,2$ & $-0,2$ & $-0,1$ \\
\hline \multirow{2}{*}{ Guyane } & Kourou & $-0,8$ & $-0,5$ & 0 & $-0,5$ & $-0,8$ \\
\hline & Ile de Cayenne & $-0,5$ & $-0,3$ & 0 & $-0,2$ & $-2,5$ \\
\hline \multirow{4}{*}{ La Réunion } & Pointe des Jardins - Port de la Marine & $-0,2$ & $-0,2$ & 0 & 0 & 0 \\
\hline & Port de la Marine - Pointe de Langevin & $-0,2$ & $-0,1$ & 0 & $-0,2$ & 0 \\
\hline & Pointe de Langevin - Pointe au Sel & $-0,4$ & $-0,5$ & 0 & $-0,1$ & 0 \\
\hline & Pointe au Sel - Pointe des Jardins & $-0,2$ & $-0,2$ & 0 & $-0,2$ & 0 \\
\hline \multirow{3}{*}{ Mayotte } & Petite Terre & $-0,1$ & $-0,1$ & 0 & $-0,1$ & 0 \\
\hline & Rassi Douamounyo - Rassi Chodoni & $-0,3$ & $-0,3$ & 0 & $-0,2$ & $-0,3$ \\
\hline & Rassi Chodoni - Rassi Douamounyo & $-0,6$ & $-0,1$ & 0 & $-0,2$ & $-0,6$ \\
\hline
\end{tabular}

\section{Scénarios d'évolution étudiés}

Les traitements réalisés fournissent une vision rétrospective de l'évolution du trait de côte sur l'ensemble des segments étudié à partir de l'exploitation de l'indicateur national de l'érosion côtière. Cette évolution tendancielle ne saurait, à elle seule, constituer une évolution plausible du trait de côte. Afin de nuancer l'approche, différentes hypothèses sont prises quant aux taux d'évolution et au devenir des aménagements côtiers. Les positions du trait de côte sont alors définies annuellement jusqu'en 2100 selon les six scénarios résultant de la combinaison de deux hypothèses sur les taux d'évolution et de trois hypothèses sur le devenir des ouvrages littoraux.

Le taux d'évolution historique calculé par l'indicateur national de l'érosion côtière est appliqué uniquement sur les secteurs identifiés en recul, soit environ $20 \%$ du trait de côte actuel. Pour ces secteurs, ce taux est reconduit et appliqué jusqu'en 2100 pour cette première hypothèse ( $\mathrm{S} 1)$. Une seconde hypothèse de recul (S2) est formulée en considérant que l'ensemble du littoral est susceptible de reculer à l'avenir sous l'effet des 


\section{Thème 7 - Risques côtiers}

conséquences attendues de l'élévation du niveau marin : les taux médians régionaux de recul sont alors pris en compte et appliqués jusqu'en 2100 (les taux de l'hypothèse S1 sont néanmoins conversés s'ils sont supérieurs aux taux dits "régionaux").

Deux hypothèses extrêmes sont formulées sur le devenir des ouvrages littoraux : les ouvrages sont soit considérés comme résistants jusqu'en 2100 (A), soit comme effacés dès à présent $(\mathrm{C})$. Une troisième hypothèse intermédiaire les complète en envisageant une destruction progressive des ouvrages à partir de leurs extrémités (B).

\section{Les enjeux étudiés}

Les données foncières (CEREMA, 2019) ont été exploitées pour cette étude. Celles-ci contiennent de très nombreuses informations sur les logements et sur le montant des transactions immobilières. Un prix médian est calculé pour les maisons et les appartements, si possible en distinguant l'âge de la construction au moment de la vente (constructions de moins d'un an et celles plus anciennes) et à la commune. Le nombre de ventes n'étant pas toujours suffisant pour atteindre un seuil statistique fiable, des regroupements de catégories de biens et des élargissements de périmètre de calcul ont été opérés pour garantir un minimum de 11 transactions dans le calcul des taux médians.

\section{Résultats}

Les positions annuelles du trait de côte sont calculées jusqu'en 2100 pour les 6 scénarios étudiés. Elles permettent d'estimer pour chaque bâtiment référencé, et chaque scénario, l'année théorique où il devrait être atteint par le recul du rivage. Selon les scénarios étudiés, de 5000 à 47300 logements (maison + appartements) pourraient être atteints par le recul du trait de côte à l'échéance 2100 (tableau 3). Les ordres de grandeur financiers correspondant à la valeur actuelle des logements concernés sont estimés entre 800 millions et 8 milliards d'euros selon le scénario considéré (tableau 3). Cette estimation économique ne prend pas en compte l'actualisation des coûts des logements, ni le département de Mayotte (où ces données sont indisponibles).

Tableau 3. Nombre et valeur ( $€$ 2018) des logements impactés par le recul des côtes.

\begin{tabular}{|c|c|c|c|}
\hline Scénarios et hypothèses & $\begin{array}{l}\text { Ouvrages maintenus en } \\
\text { place (A) }\end{array}$ & $\begin{array}{l}\text { Disparition progressive des } \\
\text { ouvrages (B) }\end{array}$ & Ouvrages effacés (C) \\
\hline $\begin{array}{l}\text { Recul au droit des secteurs } \\
\text { historiquement en érosion } \\
\text { (S1) }\end{array}$ & $\begin{array}{c}\boldsymbol{S 1 - A} \\
5000 \text { logements } \\
- \\
0,8 \mathrm{Mrd} €\end{array}$ & $\begin{array}{c}\boldsymbol{S 1 - B} \\
7600 \text { logements } \\
- \\
1,2 \mathrm{Mrd} €\end{array}$ & $\begin{array}{c}\boldsymbol{S 1 - C} \\
13300 \text { logements } \\
- \\
2,2 \mathrm{Mrd} €\end{array}$ \\
\hline $\begin{array}{l}\text { Recul sur la majorité du } \\
\text { littoral (S2) }\end{array}$ & $\begin{array}{c}\mathbf{S} \mathbf{2}-\boldsymbol{A} \\
13200 \text { logements } \\
- \\
2,2 \mathrm{Mrd} €\end{array}$ & $\begin{array}{c}\boldsymbol{S} 2-\boldsymbol{B} \\
21300 \text { logements } \\
- \\
\text { 3,7 Mrd } €\end{array}$ & $\begin{array}{c}\text { S2-C } \\
43700 \text { logements } \\
- \\
8,0 \mathrm{Mrd} €\end{array}$ \\
\hline
\end{tabular}




\section{XVIèmes Journées Nationales Génie Côtier - Génie Civil \\ Le Havre 2020}

L'évolution dans le temps de la valeur des logements impactés par le recul du trait de côte peut aussi être étudiée (figure 1) montre la forme croissante que suit leur valeur cumulée au cours de ce siècle pour chaque scénario. Compte tenu des hypothèses de l'étude, les scénarios les plus bas sont les plus réalistes à court terme mais à long terme, les scénarios élevés deviennent envisageables.

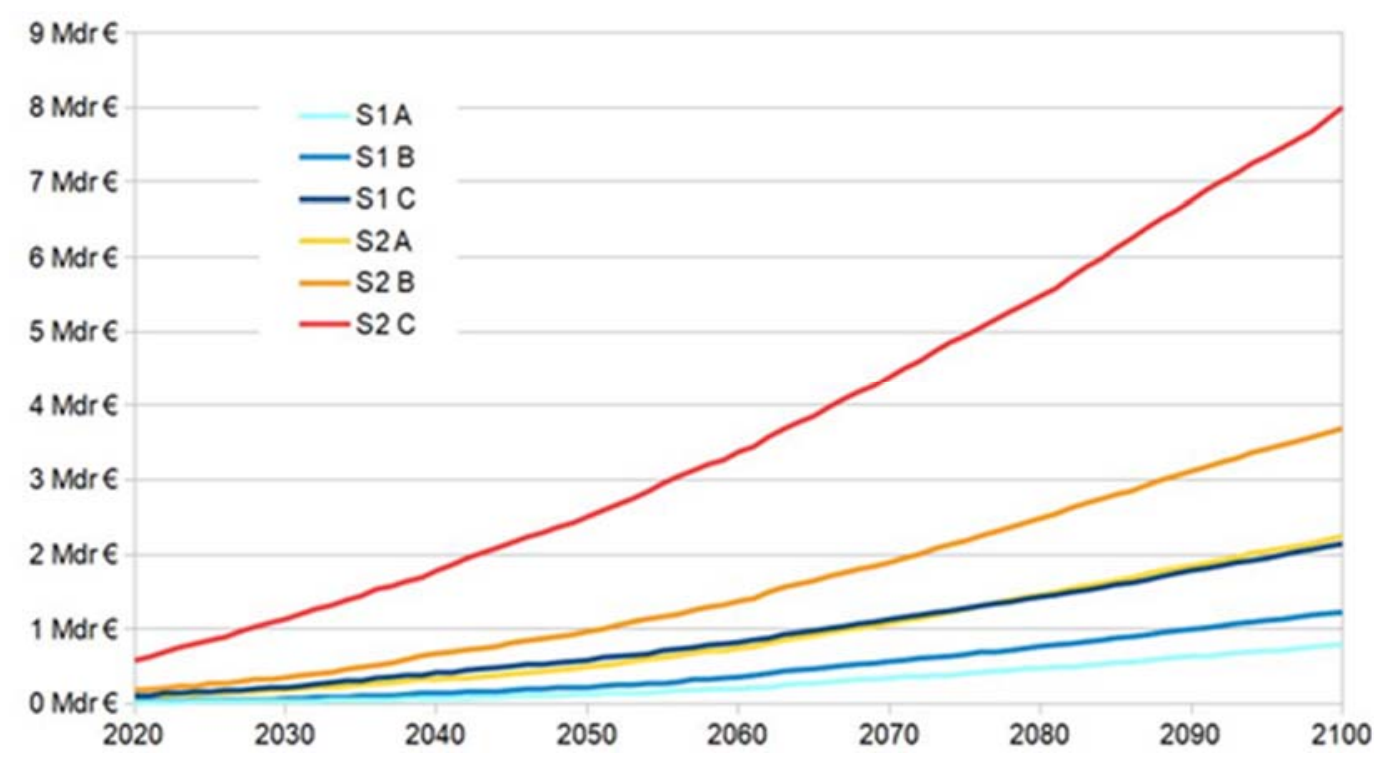

Figure 1. Evolution de la valeur des logements (€ 2018).

\section{Conclusion et discussion sur l'interprétation des résultats}

L'exploitation des connaissances nationales et homogènes apportées par la mise en œuvre des actions de la Stratégie nationale de gestion intégrée du trait de côte (SNGITC) et par les nouvelles informations foncières permettent d'estimer le nombre et la valeur des logements potentiellement impactés par le recul du trait de côte en France. Les résultats de cette étude résultent néanmoins d'hypothèses simplificatrices et de l'exploitation de données possédant des incertitudes. Même s'ils peuvent sembler précis, les résultats constituent uniquement un ordre de grandeur à l'échelle nationale des possibles conséquences du recul du trait de côte. Leur interprétation est donc à manier avec beaucoup de précautions et doit tenir compte notamment des éléments suivants. Les taux d'évolution historiques utilisés sont sujets à des incertitudes et ne permettent pas de prendre en considération d'éventuels changements de dynamique liés aux modifications des environnements côtiers. Les effets de la hausse du niveau marin sur le recul du trait de côte, associés au changement climatique, encore mal caractérisés, ne peuvent pas être pris en compte directement dans cette étude, mais sont néanmoins approchés par une projection de recul généralisé du littoral (S2). Les hypothèses sur le comportement des ouvrages côtiers (maintien / disparition progressive / effacement) sont très schématiques et ne tiennent pas compte de leurs états. Les phénomènes de submersion permanente 


\section{Thème 7 - Risques côtiers}

potentiellement induits par les effets de l'érosion sur les environnements littoraux (disparition de cordons dunaires ou d'autres points hauts, au droit de zones basses littorales) ne sont également pas pris en compte. Les enjeux et leurs valeurs sont considérés comme constants et ne tiennent pas compte des dynamiques d'aménagement futures. De même, la construction de nouveaux aménagements littoraux n'est également pas considérée ni le coût représenté par leur entretien (scénario A). Les zones portuaires abritées par des jetées, n'ont pas été étudiées dans le cadre de la cartographie de l'indicateur national de l'érosion côtière. La détermination des positions futures du trait de côte ne peut donc pas être menée dans ces secteurs particuliers.

\section{Références}

CE - Commission Européenne. (2004). Living with coastal erosion in Europe: sediment and space for sustainability. Office for Official Publications of the European Communities, Luxembourg. Part I "Major findings and Policy Recommendations of the EUROSION project", 10/05/2004, 54 p.; Part II "Maps and statistics", 29/05/2004, 25 p. CEREMA - Centre d'études et d'expertise sur les risques, l'environnement, la mobilité et l'aménagement - MTES - Ministère de la Transition écologique et solidaire. (2017). Cartographie nationale des ouvrages et aménagements littoraux. http://www.geolittoral.developpement-durable.gouv.fr/cartographie-nationale-des-ouvrages-et-r502.html

CEREMA - Centre d'études et d'expertise sur les risques, l'environnement, la mobilité et l'aménagement - MTES - Ministère de la Transition écologique et solidaire. (2018). Cartographie de l'indicateur national de l'érosion côtière. http://www.geolittoral.developpementdurable.gouv.fr/indicateur-national-de-l-erosion-cotiere-r473.html

CEREMA - Centre d'études et d'expertise sur les risques, l'environnement, la mobilité et l'aménagement. (2019). Notice relative à l'utilisation des données DV3F. https://datafoncier.cerema.fr/ressources/

HEDOU F., ROCHE A., TRMAL C., MORAUD S., DENIAUD, Y. (2018). Élaboration de l'indicateur national de l'érosion côtière. XV ${ }^{\text {ème }}$ Journées Nationales Génie Côtier Génie Civil, La Rochelle. https://doi.org/10.5150/jngcgc.2018.075

MEEM - Ministère de l'Environnement, de l’Énergie et de la Mer. (2016). Développer la connaissance et l'observation du trait de côte - Contribution nationale pour une gestion intégrée. Publication du Ministère chargé de l'écologie, en mars 2016. http://www.geolittoral.developpement-durable.gouv.fr/IMG/pdf/synthese_nationale_connaissance_trait-decote_fev_2016.pdf 